$11-1-2003$

\title{
Test Of Homogeneity For Umbrella Alternatives In Dose-Response Relationship For Poisson Variables
}

Chengjie Xiong

Washington University in St.Louis, chengjie@wubios.wustl.edu

Yan Yan

Washington University of St. Louis

Ming Ji

San Diego State University

Follow this and additional works at: http://digitalcommons.wayne.edu/jmasm

Part of the Applied Statistics Commons, Social and Behavioral Sciences Commons, and the Statistical Theory Commons

\section{Recommended Citation}

Xiong, Chengjie; Yan, Yan; and Ji, Ming (2003) "Test Of Homogeneity For Umbrella Alternatives In Dose-Response Relationship For Poisson Variables," Journal of Modern Applied Statistical Methods: Vol. 2 : Iss. 2 , Article 12.

DOI: $10.22237 /$ jmasm/1067645520

Available at: http://digitalcommons.wayne.edu/jmasm/vol2/iss2/12 


\section{Test Of Homogeneity For Umbrella Alternatives In Dose-Response Relationship For Poisson Variables}

\author{
Chengjie Xiong \\ Division of Biostatistics \\ Washington University in St. Louis
}

\author{
Yan Yan \\ Division of Biostatistics \\ Department of Surgery \\ Washington University in St. Louis \\ Ming Ji \\ Graduate School of Public Health \\ San Diego State University
}

This article concerns the testing and estimation of a dose-response effect in medical studies. We study the statistical test of homogeneity against umbrella alternatives in a sequence of Poisson distributions associated with an ordered dose variable. We propose a test similar to Cochran-Armitage's trend test and study the asymptotic null distribution and the power of the test. We also propose an estimator to the vertex point when the umbrella pattern is confirmed and study the performance of the estimator. A real data set pertaining to the number of visible revertant colonies associated with different doses of test agents in an in vitro mutagenicity assay is used to demonstrate the test and estimation process.

Key words: $C(\alpha)$ statistic, maximum likelihood estimate, monotone trend test, Poisson distribution, vertex point

\section{Introduction}

Medical studies often evaluate treatment effects at several doses of a test drug. One usually assumes a priori, based either on past experience with the test drug or on theoretical considerations, that if there is an effect on a parameter of interest, the response is likely monotonic with dose, i.e., the effect of the drug is expected to increase or decrease monotonically with increasing dose levels. Comparing several doses with a placebo in a clinical dose study is then typically performed by one-sided many-to-one comparisons or trend tests assuming an order restriction. Monotonicity of dose-response relationship, however, is far from universal.

Instances may be found where a reversal or downturn at higher doses is likely to occur. For example, many therapies for humans become counterproductive at high doses.

Address correspondence to Chengjie Xiong, Division of Biostatistics, Campus Box 8067, Washington University in St. Louis, St. Louis, MO, 63110. Telephone: 314.362.3635. Email: chengjie@wubios.wustl.edu.
Similarly, in many in vitro mutagenicity assays, experimental organisms may succumb to toxic effects at high doses of the test agents, thereby reducing the number of organisms at risk of mutation and causing a downturn in the doseresponse curve (Collings et. al., 1981; Margolin et al., 1981). These types of non-monotonic dose-response behavior may not be caused by a random effect, but may occur due to an underlying biological mechanism. Mechanistic arguments for non-monotonic dose-response shapes can be found in many medical areas, such as toxicology (Calabrese \& Baldwin, 1998), carcinogenesis (Portier \& Ye, 1998), and epidemiology (Thorogood et al., 1993).

One of the simplest non-monotonic dose-response is the so-called umbrella pattern in which the response increases (decreases) until certain dose level (usually unknown) and then decreases (increases). Ames, McCann and Yamasaki (1975) reported experimental data exhibiting this pattern from three replicate Ames tests in which plates containing Salmonella bacteria of strain TA98 were exposed to various doses of Acid Red 114. The number of visible revertant colonies on each plate was observed. Figure 1 is a scatter plot of the number of visible revertant colonies against dose level, which 
clearly indicates an umbrella pattern peaked between the third dose and the fourth dose. This same phenomenon is also observed and discussed by Simpson and Margolin (1986).

When the dose-response curve contains an umbrella pattern, the usual statistical trend tests become inadequate because of their power loss and inherent, and possibly erroneous decisions (Collings et al., 1981; Bretz \& Hothorn, 2001). The statistical test of homogeneity in response against an umbrella alternative has been studied by many authors. Most of these discussions deal with a continuous response variable and assume the normality for the associated distributions. The typical approaches under the assumption of normality are based on the framework of one-way analysis of variance and the simultaneous confidence intervals for umbrella contrasts of mean parameters (Bretz \& Hothorn, 2001; Rom et al., 1994; Shi, 1988; Marcus \& Genizi, 1994; Hayter \& Liu, 1999). Nonparametric approaches have also been considered by several authors (Lim \& Wolfe, 1997; Mack \& Wolfe, 1981 \& 1982, Simpson \& Margolin, 1994).

When data are based on counts such as those reported by Ames, McCann and Yamasaki (1975), however, a more reasonable distributional assumption might be the Poisson distribution. The statistical test of homogeneity against umbrella alternatives in a sequence of Poisson distributions associated with an ordered dose variable has not been addressed in the biostatistics literature to the best of our knowledge. This article studies this problem using an approach based on so-called $C(\alpha)$ statistics as proposed and studied by Neyman (1959) and Bailey (1956). The $C(\alpha)$ statistics are also discussed in more details by Moran (1970) and by Cox and Hinkley (1974) under the more general category of score statistics.

We propose a test similar to the Cochran-Armitage trend test and study the asymptotic null distribution and the power of our test. We also propose an estimator of the vertex point when the umbrella pattern is confirmed and study the performance of the estimator. A real data set reported by Ames, McCann and Yamasaki (1975) pertaining to the number of visible revertant colonies associated with different doses of test agents in an in vitro mutagenicity assay is used to demonstrate the test and estimation process. We also present results of a simulation study about the proposed test and estimation.

\section{Methodology}

We consider an experiment in which independent random samples are taken from $k$ distinct dose levels. Suppose that the $k$ dose levels are meaningfully ordered. Let $d_{1}, d_{2}, \ldots, d_{k}$ be the scores associated with these dose levels and $d_{1} \leq d_{2} \leq \ldots \leq d_{k}$. We assume that at dose level $i$, the response follows a Poisson distribution with mean $\mu_{i}, i=1,2, \ldots, k$. Let $n_{i}$ be the sample size associated with dose level $i$ and $n=\sum_{i=1}^{k} n_{i}$. Let $x_{i}$ be the total response in the $i$-th dose level. For each $i$ and $p, \quad 1 \leq i, p \leq k$, let $d_{i}^{p}=\left(d_{i}-d_{p}\right)^{2}$ and $\bar{d}^{p}=\sum_{i=1}^{k} n_{i} d_{i}^{p} / n$. Suppose that the relationship between the mean response and the score takes the form of

$$
\mu_{i}=H\left\lfloor\alpha+\beta\left(d_{i}-d_{p}\right)^{2}\right\rfloor,
$$

where $H$ is a monotonic function that is twice differentiable, $d_{p}$ is the dose level associated with the vertex dose of the umbrella pattern. Notice that when $p=1$ or $k$, this formulation reduces to the monotone trend. We consider the problem of testing $H_{0}: \beta=0$ against the alternative hypothesis $H_{a}: \beta \neq 0$. The likelihood function as a function of $\alpha, \beta$, and $p$ is:

$$
\begin{aligned}
& L(\alpha, \beta, p) \propto \Pi_{i=1}^{k} \\
& \exp \left(-n_{i} H\left[\alpha+\beta\left(d_{i}-d_{p}\right)^{2}\right]\right\}\left[H\left[\alpha+\beta\left(d_{i}-d_{p}\right)^{2}\right]\right]^{x_{i}} .
\end{aligned}
$$




\section{$p$ Is Known}

When $p$ is given, the test is the same as the trend test based on the redefined dose score $d_{i}^{p}, i=1,2, \ldots, k$. The test is based on the $C(\alpha)$ statistic (Moran, 1970) and is obtained by evaluating the derivative of the loglikelihood with respect to $\beta$ at the maximum likelihood estimate of $\alpha$ under $H_{0}$ :

$$
C(\alpha)=\left.\frac{\partial \log L}{\partial \beta}\right|_{\hat{\alpha}, \beta=0}=\frac{H^{\prime}(\hat{\alpha})}{H(\hat{\alpha})}\left(\sum_{i=1}^{k} x_{i} d_{i}^{p}-\hat{x} \sum_{i=1}^{k} n_{i} d_{i}^{p}\right),
$$

where

$$
\hat{x}=\frac{\sum_{i=1}^{k} x_{i}}{n},
$$

and $\hat{\alpha}=H^{-1}(\hat{x})$. The test statistic for testing $H_{0}: \beta=0$ against the alternative hypothesis $H_{a}: \beta \neq 0$ is obtained after dividing $C(\alpha)$ by its asymptotic standard deviation under $H_{0}$ computed from the information matrix of $(\alpha, \beta)$ (Tarone, 1982):

$$
X_{p}^{2}=\frac{\left[\sum_{i=1}^{k} x_{i} d_{i}^{p}-\hat{x} \sum_{i=1}^{k} n_{i} d_{i}^{p}\right]^{2}}{\hat{x} \sum_{i=1}^{k} n_{i}\left(d_{i}^{p}-\bar{d}^{p}\right)^{2}} .
$$

Notice that this test statistic does not depend on the choice of the function $H$. Under the null hypothesis, $X_{p}^{2}$ has an asymptotic Chi-square distribution with one degree of freedom. Notice also that this test statistic is identical in formula to the test statistic for testing monotone trend with the redefined score in binomial proportions proposed by Armirage (1955). In addition, Tarone (1982) showed that, like the binomial trend test (Tarone \& Gart, 1980), this Poisson trend test is asymptotically locally optimal against any choice of smooth monotone function $H$ that satisfies

$$
\mu_{i}=H\left\lfloor\alpha+\beta\left(d_{i}-d_{p}\right)^{2}\right], i=1,2, \ldots, k .
$$

\section{$p$ Is Unknown}

When $p$ is unknown and $H_{0}: \beta=0$ is tested against the alternative hypothesis $H_{a}: \beta \neq 0$, we propose to reject $H_{0}: \beta=0$ when $X^{2}=\max _{1 \leq p \leq k} X_{p}^{2}$ is large. Let $\lambda_{i}=\lim _{n \rightarrow \infty} \frac{n_{i}}{n}$ and assume that $0<\lambda_{i}<1$ for $1 \leq i \leq k$. For $1 \leq p \leq k$, let $d^{p}=\sum_{i=1}^{k} \lambda_{i} d_{i}^{p}$ and $\mu=\sum_{i=1}^{k} \lambda_{i} \mu_{i}$. Let $\Delta$ be the $k$ by $k$ matrix whose $(i, p)$ entry is given by

$$
\Delta_{i}^{p}=\frac{d_{i}^{p}-d^{p}}{\sqrt{\mu \sum_{i=1}^{k} \lambda_{i}\left(d_{i}^{p}-d^{p}\right)^{2}}} .
$$

Let $A=\left(a_{i j}\right)$ be the $k$ by $k$ matrix such that $a_{i j}=0$ if $i \neq j$ and $a_{i j}=\mu_{i} \lambda_{i}$ if $i=j$. The following theorem gives the limiting distribution of the proposed test when the null hypothesis is true.

Theorem 1: If $H_{0}$ is true, then for any $x>0$,

$$
\begin{aligned}
& \lim _{n \rightarrow \infty} P\left(X^{2} \geq x^{2}\right)= \\
& 1-\int_{-x}^{x} \int_{-x}^{x} \ldots \int_{-x}^{x} \frac{1}{\sqrt{(2 \pi)^{k}\left|\Delta^{\prime} A \Delta\right|}} \\
& \exp \left[-\frac{X^{\prime}\left(\Delta^{\prime} A \Delta\right)^{-1} X}{2}\right] d x_{1} d x_{2} \ldots d x_{k},
\end{aligned}
$$

where $X=\left(x_{1}, x_{2}, \ldots, x_{k}\right)^{\prime}$ and || is the matrix determinant.

The proof of Theorem 1 can be found in Appendix. Notice that the asymptotic null distribution does not depend on the unknown common mean $\mu_{1}=\mu_{2}=\ldots=\mu_{k}$ as the common mean $\mu$ is cancelled out in the integrand. Therefore, $\mu=1$ can always be assumed for the computation. The evaluation of 
the integration can be done by the iterative algorithm proposed by Genz (1992). This algorithm begins with a Cholesky decomposition of the covariance matrix and then uses a simple Monte-Carlo algorithm. Another possible way of evaluating the distribution of the test statistic under the null hypothesis is through a large simulation of the test statistic. We point out that the asymptotic null distribution does depend on the unknown proportion $\lambda_{i}, i=1,2, \ldots, k . \frac{n_{i}}{n}$ can be used for $\lambda_{i}$ in the computation based on the consistency of $\frac{n_{i}}{n}$ to $\lambda_{i}$. In addition, according to Šidák and Zbyněk (1967), under $H_{0}$,

$$
\operatorname{Pr}\left(X^{2} \leq x^{2}\right) \geq[2 \Phi(x)-1]^{k}
$$

where $\Phi$ is the distribution function of the standard normal distribution. Therefore, under $H_{0}$,

$$
\lim _{n \rightarrow \infty} \operatorname{Pr}\left(X^{2} \geq x^{2}\right) \leq 1-[2 \Phi(x)-1]^{k}
$$

which then provides a conservative test of $H_{0}$ against $H_{a}$.

\section{Estimation of the Vertex Point}

If the alternative hypothesis is true, the problem of interest is then the estimation of the true vertex point. To avoid the problem of parameter identification, we assume that the umbrella pattern satisfies

$$
\mu_{1} \leq \mu_{2} \leq \ldots \leq \mu_{l-1}<\mu_{l}>\mu_{l+1} \geq \ldots \geq \mu_{k}
$$

or

$$
\mu_{1} \geq \mu_{2} \geq \ldots \geq \mu_{l-1}>\mu_{l}<\mu_{l+1} \leq \ldots \leq \mu_{k},
$$

i.e., we only consider the case where a single vertex point $l$ exists. Notice that this formulation does not rule out the possibility that the vertex point is on the boundary of the dose interval if a monotone trend is the alternative hypothesis. We propose to estimate $l$ by $\hat{l}$ such that $X_{\hat{l}}^{2}=\max _{1 \leq p \leq k} X_{p}^{2}$, where $X_{p}^{2}$ is given by (1). Notice that as $n \rightarrow \infty$, for any $1 \leq p \leq k$,

$$
\begin{aligned}
\lim _{n \rightarrow \infty} \frac{X_{p}^{2}}{n}= & \frac{\left[\sum_{i=1}^{k} \lambda_{i} \mu_{i} d_{i}^{p}-\sum_{i=1}^{k} \lambda_{i} \mu_{i} \sum_{i=1}^{k} \lambda_{i} d_{i}^{p}\right]^{2}}{\sum_{i=1}^{k} \lambda_{i} \mu_{i} \sum_{i=1}^{k} \lambda_{i}\left(d_{i}^{p}-d^{p}\right)^{2}} \\
& =\frac{\sum_{i=1}^{k} \lambda_{i}\left(\mu_{i}-\mu\right)^{2}}{\sum_{i=1}^{k} \lambda_{i} \mu_{i}} R_{U^{p}, V}^{2},
\end{aligned}
$$

where $R_{U^{p}, V}^{2}$ is the correlation coefficient between random variables $U^{p}$ and $V$ defined on the sample space $\{1,2, \ldots, k\}$ with the multinomial probability distribution $\left\{\lambda_{1}, \lambda_{2}, \ldots, \lambda_{k}\right\}$, and $U^{p}(i)=d_{i}^{p}, V(i)=\mu_{i}$. Since

$$
\begin{aligned}
& -U^{p}(1) \leq-U^{p}(2) \leq \ldots \leq-U^{p}(p-1)<-U^{p}(p) \\
& =0>-U^{p}(p+1) \geq \ldots \geq-U^{p}(k)
\end{aligned}
$$

and either

$$
\mu_{1} \leq \mu_{2} \leq \ldots \leq \mu_{l-1}<\mu_{l}>\mu_{l+1} \geq \ldots \geq \mu_{k}
$$

or

$$
\mu_{1} \geq \mu_{2} \geq \ldots \geq \mu_{l-1}>\mu_{l}<\mu_{l+1} \leq \ldots \leq \mu_{k}
$$

holds, the proposed estimator to the true vertex point $l$ asymptotically maximizes the square of the correlation coefficient between $U^{p}$ and $V$ over $p=1,2, \ldots, k$.

\section{A Real Example}

In in vitro mutagenicity assays, experimental organisms may succumb to toxic effects at high doses of test agents, thereby reducing the number of organisms at risk of 
mutation and causing a downturn in the doseresponse curve (Collings et al., 1981; Margolin et al., 1981).

Ames, McCann and Yamasaki (1975) reported experimental data exhibiting this pattern from three replicate Ames tests in which plates containing Salmonella bacteria of strain TA98 were exposed to various doses of Acid Red 114. The number of visible revertant colonies on each plate was observed. We assume a Poisson distribution for the number of visible revertant colonies and test whether an umbrella pattern in the mean exists.

Figure 1 is a scatter plot of the number of visible revertant colonies against dose level, which clearly indicates an umbrella pattern peaked between the third dose and the fourth dose. The test statistic is

$$
\begin{aligned}
X^{2} & =\max (75.71,75.76,75.90,76.20,69.78,55.96) \\
& =76.20 .
\end{aligned}
$$

The conservative test gives a $p$-value less than 0.00001, indicating a strong evidence that an umbrella pattern exists. Since $\max _{1 \leq p \leq 6} X_{p}^{2}$ is obtained when $p=4$, i.e., when dose $d_{4}=10000(\mu \mathrm{g} / \mathrm{ml})$ of Acid Red 114 is used, the estimated peak dose is $d_{4}=10000$ $(\mu \mathrm{g} / \mathrm{ml})$.

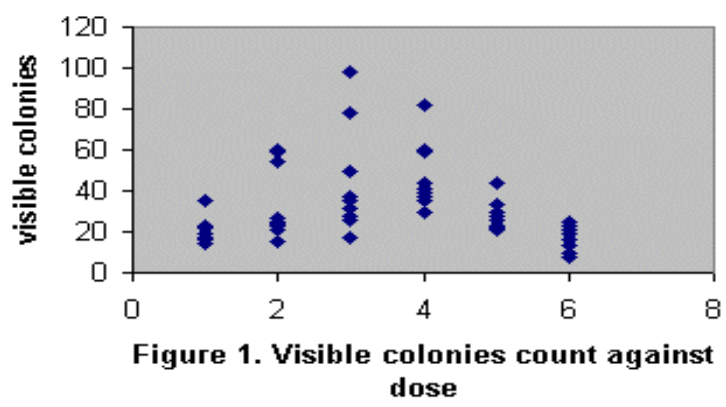

Simulation Studies

To understand the performance of the proposed test and the estimator for the vertex point when the alternative hypothesis is true, we have carried out a simulation study to evaluate the statistical power of the proposed test and the probability that the vertex point estimator correctly estimates the true vertex point for a set of selected parameters.

In our simulation, we assume that a total of five independent Poisson distributions associated with five different dose levels $d_{i}=i, i=0,1,2,3,4$. We also assume that the sample size of all 5 groups is the same, i.e., $n_{1}=n_{2}=n_{3}=n_{4}=n_{5}$. Theorem 1 is used to determine the $x^{2}$ which achieves the upper $5 \%$ percentile of the test statistic under the null hypothesis.

The empirical power of the proposed test is computed as the proportion of rejections of the null hypothesis over repeated independent tests with a selected set of umbrella patterns. The performance of the proposed estimator to the vertex point is assessed by computing the empirical probability that the proposed estimator correctly estimates the true vertex point.

Table 1 presents the empirical power of the test and the empirical probability of correct estimation of the vertex point for three different choices of the true umbrella pattern and various sample sizes. Each entry in Table 1 is computed from 10000 independent hypotheses tests and estimations. All the tests assume a significance level of $5 \%$.

The first column in Table 1 is the true mean vector $\left(\mu_{1}, \mu_{2}, \mu_{3}, \mu_{4}, \mu_{5}\right)$. Notice that these umbrella patterns are chosen so that each possible interior vertex point (i.e., $l=2,3,4$ ) within the boundary of the dose interval is considered. Because the monotone trend is included in the alternative hypothesis when the vertex point falls on the boundary of the dose interval, it is of interest to see how our proposed test performs in these alternatives.

This is relevant given the fact that, when an umbrella pattern is likely in the dose-response relationship, the traditional statistical monotone trend tests become inadequate because of their power loss and inherent, and possibly erroneous decisions (Collings et al., 1981; Bretz and Hothorn, 2001). We simulated the statistical power of the proposed test for detecting the monotone trend and compared that to the traditional trend test as discussed by Cochran (1954) and Tarone (1982). 
Table 1: Empirical Power and Probability with an Interior Vertex Point.

\begin{tabular}{|c|c|c|c|}
\hline Umbrella Pattern & $\begin{array}{c}\text { Sample Size Per } \\
\text { Dose }\end{array}$ & Power (\%) & $\begin{array}{l}\text { Correct Vertex } \\
\text { Estimation (\%) }\end{array}$ \\
\hline \multirow[t]{6}{*}{$(2,2.5,3,2.5,1.5)$} & 10 & 51.98 & 68.81 \\
\hline & 20 & 84.66 & 77.90 \\
\hline & 30 & 96.56 & 82.85 \\
\hline & 40 & 99.22 & 87.18 \\
\hline & 50 & 99.79 & 89.71 \\
\hline & 80 & 100 & 93.59 \\
\hline \multirow[t]{6}{*}{$(1.5,2,2.5,3,2.5)$} & 10 & 53.31 & 47.84 \\
\hline & 20 & 85.97 & 57.63 \\
\hline & 30 & 96.54 & 62.92 \\
\hline & 40 & 99.34 & 66.64 \\
\hline & 50 & 99.86 & 69.15 \\
\hline & 80 & 100 & 74.32 \\
\hline \multirow[t]{6}{*}{$(2.5,3,2.5,2,1.5)$} & 10 & 53.23 & 46.85 \\
\hline & 20 & 85.47 & 58.08 \\
\hline & 30 & 96.60 & 63.88 \\
\hline & 40 & 99.24 & 66.64 \\
\hline & 50 & 99.79 & 68.66 \\
\hline & 80 & 100 & 74.09 \\
\hline
\end{tabular}


Table 2 provides the empirical power and the comparison along with the empirical probability of the correct estimation of the vertex point. The second column in Table 2 is the empirical power based on our proposed test. The third column in Table 2 is the empirical power based on the test by Cochran (1954) and Tarone (1982). Because the vertex point for a monotone trend could be either $l=1$ or $l=5$, the
Another different type of alternative hypothesis is when a flat segment appears in the Poisson mean vector $\left(\mu_{1}, \mu_{2}, \mu_{3}, \mu_{4}, \mu_{5}\right)$.

Table 3 presents the empirical power and the empirical probability of the correct estimation of the vertex points for several different choices of such patterns. Since the vertex point in some of these situations is not unique, the empirical probability of the correct

\section{Table 2: Empirical Power and Probability with a Boundary Vertex Point.}

$\begin{array}{ccccc}\text { Umbrella Pattern } & \begin{array}{c}\text { Sample Size } \\ \text { Per Dose }\end{array} & \text { Power }^{1}(\%) & \text { Power }^{2}(\%) & \begin{array}{c}\text { Correct Vertex } \\ \text { Estimation }(\%)\end{array} \\ (1.5,1.8,2.0,2.3,2.5) & 10 & 33.69 & 41.85 & 52.86 \\ & 20 & 62.45 & 70.45 & 68.05 \\ & 30 & 80.55 & 86.65 & 78.25 \\ & 40 & 90.20 & 94.18 & 84.46 \\ (30 & 95.58 & 97.45 & 88.14 \\ & 80 & 99.71 & 99.89 & 94.91 \\ & 10 & 22.81 & 28.27 & 44.66 \\ & 20 & 40.99 & 49.29 & 57.09 \\ & 30 & 56.80 & 65.14 & 66.32 \\ & 40 & 70.90 & 78.63 & 73.20 \\ & 50 & 80.05 & 86.83 & 78.59 \\ & 50 & 94.85 & 97.14 & 88.04\end{array}$

${ }^{1}$ Proposed test, ${ }^{2}$ Cochran \& Tarone's test.

empirical probability of the correct estimation to the true vertex points reported in Table 2 refers to the proportion over repeated estimates that either $l=1$ or $l=5$ is correctly estimated. Each entry in Table 2 is also computed from 10000 independent hypotheses tests and estimations. estimation reported in Table 3 refers to the proportion that one of the possible vertex points is correctly identified over 10000 independent estimates. Data simulations are done using the random number generating function RANPOI 
Table 3: Empirical Power and Probability with a Flat Segment in the Pattern

\begin{tabular}{|c|c|c|c|}
\hline Umbrella Pattern & $\begin{array}{c}\text { Sample Size Per } \\
\text { Dose }\end{array}$ & Power (\%) & $\begin{array}{l}\text { Correct Vertex } \\
\text { Estimation }(\%)\end{array}$ \\
\hline \multirow[t]{6}{*}{$(2.5,3.0,3.0,2.5,2.0)$} & 10 & 26.18 & 77.21 \\
\hline & 20 & 50.48 & 87.36 \\
\hline & 30 & 69.76 & 92.19 \\
\hline & 40 & 82.76 & 95.12 \\
\hline & 50 & 90.61 & 96.64 \\
\hline & 80 & 98.95 & 98.91 \\
\hline \multirow[t]{6}{*}{$(2 \cdot 5,3 \cdot 0,3 \cdot 0,3 \cdot 0,2.5)$} & 10 & 11.95 & 83.36 \\
\hline & 20 & 20.81 & 90.08 \\
\hline & 30 & 30.39 & 93.72 \\
\hline & 40 & 40.36 & 95.98 \\
\hline & 50 & 49.78 & 97.37 \\
\hline & 80 & 71.38 & 99.36 \\
\hline \multirow[t]{6}{*}{$(2.5,2.5,3.0,3.0,2.5)$} & 10 & 9.71 & 63.19 \\
\hline & 20 & 15.07 & 70.92 \\
\hline & 30 & 21.47 & 78.14 \\
\hline & 40 & 28.10 & 81.78 \\
\hline & 50 & 34.82 & 85.59 \\
\hline & 80 & 52.70 & 92.83 \\
\hline
\end{tabular}


from Statistical Analysis System (SAS Institute, Inc. 1999).

\section{Conclusion}

When an umbrella pattern is likely in the doseresponse relationship, the usual statistical trend tests become inadequate because of their power loss and inherent, and possibly erroneous decisions (Collings et al., 1981; Bretz \& Hothorn, 2001). We proposed in this paper a test of homogeneity against umbrella alternatives in a sequence of Poisson distributions associated with an ordered dose variable and studied the limiting null distribution and the statistical power.

We also proposed an estimator of the vertex point when the umbrella pattern is confirmed and studied the performance of the estimator. Although the simulation study verifies that the increase of the sample size always increases the statistical power of the test and the probability of the correct estimation to the vertex point, Table 1 seems to indicate that for the selected set of parameters, the proposed estimator to the true vertex point performs better when the vertex point $(l=3)$ is in the middle of the dose interval than when it is away from the middle of the dose interval $(l=2,4)$. The statistical power of the proposed test, however, seems to be very comparable wherever the interior vertex is.

Our proposed test not only detects an umbrella pattern effectively based on the simulation results in Table 1, but also possesses reasonable statistical power to detect a monotone trend which is a subset of the alternative hypothesis considered in this paper. In fact, the simulation in Table 2 shows that, although our proposed test does not have as much the statistical power for detecting the monotone trend as the trend test of Cochran (1954), the difference in power between these two tests is relatively small. This is especially promising given the fact that the trend test of Cochran (1954) is asymptotically locally optimal against any choice of smooth monotone function $H$ (Tarone, 1982).

On the other hand, the simulation results reported in Table 3 seem to indicate that the statistical power of the proposed test deteriorates when a substantial flat segment exists in the mean vector of the Poisson distributions, although the proposed vertex estimator still shows a high probability of correctly identifying one of these multiple vertex points.

Like the similarity on the test statistic for testing a monotone trend between a sequence of binomial distributions and a sequence of Poisson distributions (Armitage 1955; Cochran 1954), the proposed test and estimation techniques can be readily extended to the situation for detecting an umbrella pattern in a sequence of binomial distributions.

\section{References}

Ames, B. N., McCann, J., \& Yamasaki, E. (1975). Methods for detecting carcinogens and mutagens with the Salmonella/Microsome Mutagenicity test. Mutation Research, 31, 347364.

Armitage, P. (1955). Tests for linear trends in proportions and frequencies. Biometrics, 11, 375-386.

Bailey, N. T. J. (1956). Significance tests for a variable chance of infection in chainbinomial theory, Biometrika, 43, 332-336.

Bretz, F., \& Hothorn, L. A. (2001). Testing dose-response relationships with a priori unknown, possibly nonmonotone shape. Journal of Biopharmaceutical Statistics, 11, 3, 193-207.

Calabrese, E. J., \& Baldwin, L. A. (1998). A general classification of U-shape dose-response relationships in Toxicology and their mechanistic foundations. Human and Experimental Toxicology, 17, 353-364.

Cochran, W. G. (1954). Some methods for strengthening the common $\chi^{2}$ tests. Biometrics, 10, 417-451.

Collings, B. J. Margolin, B. H., \& Oehlert, G. W. (1981). Analysis for binomial data, with application to the fluctuation tests for mutagenicity. Biometrics, 37, 775-794.

Cox, D. R., \& Hinkley, D. V. (1974). Theoretical Statistics, London: Chapman and Hall.

Genz, A. (1992). Numerical computation of multivariate normal probabilities. Journal of Computational and Graphical Statistics 1, 141-149. 
Hayter, A. J., \& Liu, W. (1999). A new test against an umbrella alternative and the associated simultaneous confidence intervals. Computational Statistics \& Data Analysis, 30, 393-401.

Lim, D. H., \& Wolfe, D. A. (1997). Nonparametric tests for comparing umbrella pattern treatment effects with a control in a randomized block design. Biometrics, 53, 410418.

Mack, G. A., \& Wolfe, D. A. (1981). Ksample rank tests for umbrella alternatives. Journal of the American Statistical Association 76, 175-181; Correction 1982, 53, 410-418.

Marcus, R., \& Genizi, A. (1994). Simultaneous confidence intervals for umbrella contrasts of normal means. Computational Statistics \& Data Analysis, 17, 393-407.

Margolin, B. H., Kaplan, N., \& Zeiger, E. (1981). Statistical analysis of the Ames Salmonella / Microsome test. Proceedings of the National Academy of Sciences of the United States of America, 78, 3779-3783.

Moran, P. A. P. (1970). On asymptotically optimal tests of composite hypotheses. Biometrika, 57, 47-55.

Neyman, J. (1959). Optimal asymptotic tests of composite hypotheses, in Probability and Statistics. U. Grenander (Ed.). New York: John Wiley \& Sons, 213-234.

Portier, C. J., \& Ye, F. (1998). U-shaped dose-response curves for carcinogens. Human and Experimental Toxicology, 17, 705-707.
Rom, D. M., Costello, R. J., \& Connell, L. T. (1994). On closed test procedures for doseresponse analysis. Statistics in Medicine, 13, 1583-1596.

SAS Institute, Inc. (1999). SAS Language (Version 8), Cary, NC.

Shi, N. Z. (1988). A test of homogeneity for umbrella alternatives and tables of the level probabilities. Communications in Statistics-Theory and Methods, 17, 657-670.

Šidák, Z. (1967). Rectangular confidence regions for the means of multivariate normal distributions. Journal of American Statistical Association, 62, 626-633.

Simpson, D. G., \& Margolin, B. H. (1986). Recursive nonparametric testing for dose-response relationship subject to downturns at high doses. Biometrika, 73, 3, 589-596.

Tarone, R. E. (1982). The use of historical control information in testing for a trend in Poisson means. Biometrics, 38, 457462.

Tarone R. E., \& Gart, J. J. (1980). On the robustness of combined tests for trends in proportions. Journal of American Statistical Association, 75, 110-116.

Thorogood, M., Mann, J., \& McPherson, K. (1993). Alcohol intake and the U-shaped curve: Do non-drinkers have a higher prevalence of cardiovascular-related disease? Journal of Public Health Medicine, 15, 61-68. 


\section{Appendix}

We give the proof of Theorem 1, which gives the null distribution of $X^{2}$. Let

$$
Y_{p}=\frac{\left[\sum_{i=1}^{k} \frac{x_{i} d_{i}^{p}}{n}-\hat{x} \sum_{i=1}^{k} \lambda_{i} d_{i}^{p}\right]}{\sqrt{\mu \sum_{i=1}^{k} \lambda_{i}\left(d_{i}^{p}-d^{p}\right)^{2}}}=\sum_{i=1}^{k} \frac{x_{i} \Delta_{i}^{p}}{n}
$$

where

$$
\begin{aligned}
d^{p} & =\sum_{i=1}^{k} \lambda_{i} d_{i}^{p} \\
\mu & =\sum_{i=1}^{k} \lambda_{i} \mu_{i} \\
\Delta_{i}^{p} & =\frac{d_{i}^{p}-\sum_{i=1}^{k} \lambda_{i} d_{i}^{p}}{\sqrt{\mu \sum_{i=1}^{k} \lambda_{i}\left(d_{i}^{p}-d^{p}\right)^{2}}} .
\end{aligned}
$$

Let $\hat{X}_{i}=\frac{x_{i}}{n}$. Note that

$$
\left(\begin{array}{c}
Y_{1} \\
Y_{2} \\
\cdots \\
Y_{k}
\end{array}\right)=\Delta^{\prime}\left(\begin{array}{c}
\hat{X}_{1} \\
\hat{X}_{2} \\
\cdots \\
\hat{X}_{k}
\end{array}\right)
$$

where $\Delta=\left(\Delta_{i}^{p}\right)$ is the $k$ by $k$ matrix whose $(i, p)$ entry is $\Delta_{i}^{p}$. Since

$$
\sqrt{n}\left[\left(\begin{array}{c}
\hat{X}_{1} \\
\hat{X}_{2} \\
\ldots \\
\hat{X}_{k}
\end{array}\right)-\left(\begin{array}{c}
\lambda_{1} \mu_{1} \\
\lambda_{2} \mu_{2} \\
\cdots \\
\lambda_{k} \mu_{k}
\end{array}\right)\right] \rightarrow N(0, A)
$$

where $A=\left(a_{i j}\right)$ is the $k$ by $k$ matrix such that $a_{i j}=0$ if $i \neq j$ and $a_{i j}=\mu_{i} \lambda_{i}$ if $i=j$, and the limit is in distribution. Therefore,

$$
\sqrt{n}\left[\left(\begin{array}{c}
Y_{1} \\
Y_{2} \\
\ldots \\
Y_{k}
\end{array}\right)-\Delta^{\prime}\left(\begin{array}{c}
\lambda_{1} \mu_{1} \\
\lambda_{2} \mu_{2} \\
\ldots \\
\lambda_{k} \mu_{k}
\end{array}\right)\right] \rightarrow N\left(0, \Delta^{\prime} A \Delta\right) .
$$

Theorem 1 follows from the fact that $\sqrt{n}\left(Y_{1}, Y_{2}, \ldots, Y_{k}\right)^{\prime}$ and $\left(X_{1}, X_{2}, \ldots, X_{k}\right)^{\prime}$ are stochastically equivalent under $H_{0}$. 